\title{
Shift in cytotoxic target from estrogen receptor-positive to estrogen receptor-negative breast cancer cells by trastuzumab in combination with taxane-based chemotherapy
}

\author{
MITSUHIRO HAYASHI ${ }^{1,3}$, KAZUHARU KAI $^{4}$, YASUHIRO OKUMURA ${ }^{1}$, TOMOFUMI OSAKO $^{1}$, \\ NOBUYUKI ARIMA ${ }^{2}$, HIROTAKA IWASE ${ }^{3}$ and REIKI NISHIMURA ${ }^{1}$
}

\author{
Departments of ${ }^{1}$ Breast and Endocrine Surgery, ${ }^{2}$ Clinical Pathology, Kumamoto City Hospital, \\ Kumamoto 862-8505; ${ }^{3}$ Department of Breast and Endocrine Surgery, Faculty of Life Sciences, Kumamoto University \\ Graduate School of Medical Sciences, Kumamoto 860-8556; ${ }^{4}$ Department of Gene Regulation, \\ Institute for Advanced Medical Research, Keio University School of Medicine, Shinjuku-ku, Tokyo 160-8582, Japan
}

Received September 16, 2010; Accepted December 16, 2010

DOI: $10.3892 / \mathrm{ol} .2011 .232$

\begin{abstract}
Trastuzumab has shown significant clinical benefits in patients with operable and metastatic HER2-positive breast cancer. However, the biological mechanism of the additional effect of trastuzumab administered in combination with conventional chemotherapy is poorly understood. We performed a retrospective analysis of 55 patients with HER2positive breast cancer treated with anthracycline and taxane (chemotherapy alone; CT), or trastuzumab in combination with taxane-based chemotherapy $(\mathrm{CT}+\mathrm{T})$ for neoadjuvant chemotherapy. We determined the therapeutic efficacies [clinical (CR) and pathological complete responses (pCR)] and changes in the proportion of positive cells for each biomarker pre- to post-neoadjuvant chemotherapy for each treatment regimen. Clinical-CR and quasi-pCR rates defined as the absence of invasive tumors or only a few remaining invasive tumor cells were 6.9 and $31.0 \%$ in the CT group and 46.2 and $65.4 \%$ in the $\mathrm{CT}+\mathrm{T}$ group, respectively. In the $\mathrm{CT}$ group, the proportion of estrogen receptor (ER)-/progesterone receptor (PgR)-positive cells decreased significantly following treatment (ER, 73.5 vs. $50.9 \% ; \mathrm{P}=0.02)$. Changes in the proportion of ER-/PgR-positive cells were not noted in the CT+T group (ER, 81.9 vs. $80.3 \%$; $\mathrm{P}=0.61$, although a relatively greater decrease in the proportion of $\mathrm{Ki}-67$-positive cells was found in the $\mathrm{CT}+\mathrm{T}$ group than that in the CT group (-26.5 vs. $-13.7 \%$ ). These findings indicate that CT+T inhibits ER-negative and Ki-67-positive breast cancer cells. In conclusion, trastuzumab sensitized ER-negative proliferative cells to cytotoxic chemotherapy. This finding may indicate an additional clinical effect of trastuzumab when
\end{abstract}

Correspondence to: Dr Reiki Nishimura, Department of Breast and Endocrine Surgery, Kumamoto City Hospital, 1-1-60 Kotoh, Kumamoto City, Kumamoto 862-8505, Japan

E-mail: nishimura.reiki@cityhosp-kumamoto.jp

Key words: trastuzumab, neoadjuvant therapy, estrogen receptor administered in combination with conventional chemotherapy as neoadjuvant chemotherapy for HER2-positive breast cancer.

\section{Introduction}

Trastuzumab is a well-known molecular-targeting drug which provides significant clinical benefits to patients with human epidermal growth factor (HER)2-positive breast cancer (1). In metastatic breast cancer, the efficacy of trastuzumab was proven in terms of improved objective responses, resulting in an increase in patient survival $(2,3)$. The additional or synergistic effect of trastuzumab administered in combination with conventional types of chemotherapy has been suggested to play a role in pre-operative treatment based on improvement in the objective response and pathological complete response (pCR) rates $(4,5)$.

Although the molecular mechanism underlying its action is poorly understood, evidence is available for the efficacy of trastuzumab in clinical practice. Briefly, trastuzumab directly targets the extracellular domain of HER2 and inhibits proliferation and the anti-apoptotic potential of HER2-positive breast cancer cells by down-regulating pathways, such as PI3K/Akt and MAPK (1).

However, cellular heterogeneity, such as sensitivities to cytotoxic agents, positivity for the estrogen receptor (ER) and proliferation revealed as Ki-67 expression, exists even in HER2positive cancers. Furthermore, the cancer stem cell (CSC) hypothesis, was also found to verify the existence of intrinsic cellular and molecular heterogeneity within each tumor $(6,7)$.

Despite evidence for heterogeneity or the CSC hypothesis, which HER2-positive breast cancer cells are targeted by trastuzumab or which molecular pathways are involved in the mechanism of trastuzumab activity has yet to be elucidated. To resolve this issue, a retrospective analysis of HER2-positive breast cancer patients treated with neoadjuvant chemotherapy (NAC), with or without trastuzumab, was performed. The therapeutic efficacies and proportional changes of positive cells of each biomarker pre- to post-NAC for each treatment regimen were investigated. These changes and any discrepancy 
in these changes may represent treatment-resistant tumor cells in non-responders and provide evidence of a cellular shift in target by trastuzumab administered in combination with conventional chemotherapy as NAC.

\section{Patients and methods}

Patients. A retrospective analysis of a prospectively maintained clinical database was performed, including patients who received NAC at Kumamoto City Hospital, Kumamoto City, Japan, from March 2002 to June 2009. Of the 144 patients who received NAC during this period, 55 patients with HER2-positive breast cancers were enrolled in this study. A prerequisite of the hospital's NAC inclusion criteria was invasive tumors of a diameter of $\geq 3 \mathrm{~cm}$ and/or showing lymph node involvement. All 144 patients fulfilled these criteria. The research protocol of the present study was approved by the Ethics Committee of Kumamoto City Hospital, and informed consent was obtained from all patients prior to treatment. Certain patients had also been enrolled in a prospective multicenter phase II trial conducted by the Kyushu Breast Cancer Study Group or Kumamoto Breast Cancer Study Group.

Treatment regimens. The treatment regimens used in the present study were: 18 patients were treated concurrently with epirubicin and docetaxel (ET); 11 patients were treated with 5-fluorouracil, epirubicin, and cyclophosphamide (FEC) followed by docetaxel (DOC); and 26 patients were treated with anthracycline-based chemotherapy followed by concurrent trastuzumab with taxane. All 29 patients treated with ET or FEC followed by DOC were considered to be the conventional chemotherapy (CT) group.

For the ET regimen, the patients received 4 cycles of epirubicin $\left(60 \mathrm{mg} / \mathrm{m}^{2}\right)$ and docetaxel $\left(60 \mathrm{mg} / \mathrm{m}^{2}\right)$ every 3 weeks. For the FEC followed by the DOC regimen, the patients received 4 cycles of 5 -fluorouracil $\left(500 \mathrm{mg} / \mathrm{m}^{2}\right)$, epirubicin $\left(75-100 \mathrm{mg} / \mathrm{m}^{2}\right)$ and cyclophosphamide $\left(500 \mathrm{mg} / \mathrm{m}^{2}\right)$ every 3 weeks, followed by 4 cycles of docetaxel $\left(70-80 \mathrm{mg} / \mathrm{m}^{2}\right)$ every 3 weeks. For the trastuzumab-containing regimens, the patients received 4 cycles of epirubicin $\left(90 \mathrm{mg} / \mathrm{m}^{2}\right)$ and cyclophosphamide $\left(600 \mathrm{mg} / \mathrm{m}^{2}\right)$ every 3 weeks, followed by concurrent weekly trastuzumab $(4 \mathrm{mg} / \mathrm{kg}$ on day 1 and subsequent infusions at a dose of $2 \mathrm{mg} / \mathrm{kg}$ ) with 12 cycles of weekly paclitaxel $\left(80 \mathrm{mg} / \mathrm{m}^{2}\right)$ or 4 cycles of FEC followed by concurrent weekly trastuzumab with 4 cycles of tri-weekly docetaxel.

Three to four weeks after completion of NAC, breastconserving surgery or mastectomy with sentinel node biopsy or axillary lymph node dissection was performed with intent to cure. Patients who underwent breast-conserving surgeries received radiotherapy to the conserved breast. Patients with hormone receptor (HR)-positive cancers received adjuvant endocrine therapy according to the patient's menopausal status.

Evaluation of clinical and pathological responses. Definitive diagnoses of invasive breast cancers were performed by the hospital's pathologist on hematoxylin and eosin-stained sections obtained from core needle biopsy samples. Biological markers were examined both at diagnosis and surgery using the samples of core needle biopsy and residual tumor in the operated breast, respectively. The variables of interest were tumor size, lymph node involvement, nuclear grade, proportions of ER-/progesterone receptor (PgR)-positive cells, HER2 status and Ki-67 labeling index. Immunohistochemical staining was performed according to a protocol described previously (8).

At least 5 microscopic fields (x40) were counted to determine the proportion of ER- and PgR-positive tumor cells, and the observed proportions were calculated as a percentage of all counted cells. The hormone receptor status was determined based on the dichotomic criteria as: positive if the proportion of cells was $\geq 10 \%$ and negative if the proportion was $<10 \%$. ER- and/or PgR-positive cells were rated as HR-positive. Proliferation activity was judged by immunostaining with the Ki-67 antibody (Dako, Glostrup, Denmark). The proportion of proliferating cells was determined by counting at least 500 tumor cells. HER2 expression was initially evaluated using the Hercep Test (Dako). HER2 positivity was indicated by $3+$ staining intensity. The HER 2 equivocal (2+ staining) was tested using fluorescence in situ hybridization with a threshold ratio of $>2.0$ for positive HER2:CEP17.

Tumor burden was measured by caliper and ultrasound after each cycle and evaluated by the Response Evaluation Criteria in Solid Tumors (9). The pathological responses were assessed in surgical specimens of the breast with reference to the standards of the Japanese Breast Cancer Society (10). Based on this criterion, pathological responses were evaluated based on invasive components and not carcinoma in situ. Thus, tumors with residual ductal carcinoma in situ were included in the pCR group. Furthermore, marked changes approaching a complete response in only a few remaining invasive tumor cells were classified as near-pCR (11). Patients with pCR and near-pCR were classified as pathological responders, and their pathological responses were defined as quasi-pCR (12). The remaining patients were designated as pathological nonresponders.

Evaluation of changes in the proportion of positive cells for each biomarker. To assess the biological mechanism of the additional effect of trastuzumab administered in combination with conventional types of chemotherapy, the proportion of positive cells for each biomarker was determined at diagnosis and surgery. Using immunohistochemical staining, the tumor defined as pCR was excluded from the analysis, but the tumor defined as near-pCR with evaluable cells was included. To focus on ER, PgR and $\mathrm{Ki}-67$ independently, a subgroup analysis was performed for breast tumors with $10 \%$ or higher proportional positivity for ER and PgR, which indicate HR-/ HER2-positive breast cancer.

Statistical analysis. Statistical comparisons were performed using the Chi-square test and the paired Student's t-test. A two-sided P-value of $<0.05$ was considered to be statistically significant. JMP 8.0 software package (SAS Institute Inc., Cary, NC, USA) was used for statistical analysis.

\section{Results}

Clinical and pathological responses in HER2-positive breast cancer. The patient characteristics at diagnosis are shown in Table I. No significant differences were noted in patient 
Table I. Patient characteristics at diagnosis.

\begin{tabular}{|c|c|c|c|}
\hline & $\begin{array}{l}\text { Chemotherapy alone } \\
\qquad(\mathrm{n}=29)\end{array}$ & $\begin{array}{l}\text { Chemotherapy+Trastuzumab } \\
\qquad(\mathrm{n}=26)\end{array}$ & \\
\hline Characteristics & No. of patients (\%) & No. of patients (\%) & P-value \\
\hline Age (years) & & & 0.62 \\
\hline Median & 53 & 51 & \\
\hline Range & $29-67$ & $32-67$ & \\
\hline Tumor size & & & 0.89 \\
\hline $\mathrm{T} 1$ & $2 \quad(7)$ & $2 \quad(8)$ & \\
\hline $\mathrm{T} 2$ & $15 \quad(52)$ & $14 \quad(54)$ & \\
\hline $\mathrm{T} 3$ & $10 \quad(35)$ & $7 \quad(27)$ & \\
\hline $\mathrm{T} 4$ & $2 \quad(7)$ & $3 \quad(12)$ & \\
\hline Total & $29(100)$ & $26(100)$ & \\
\hline Axillary nodes & & 0.46 & \\
\hline No & $12(41)$ & $7 \quad(27)$ & \\
\hline $\mathrm{N} 1$ & 14 (48) & $15 \quad(58)$ & \\
\hline $\mathrm{N} 2$ & 3 (10) & $3 \quad(12)$ & \\
\hline N3 & $0 \quad(0)$ & $1 \quad(4)$ & \\
\hline Total & $29(100)$ & $26(100)$ & \\
\hline Hormone receptor & & & 0.32 \\
\hline ER- and/or PgR-positive & 14 (48) & $16 \quad(62)$ & \\
\hline ER- and PgR-negative & $15 \quad(52)$ & $10 \quad(38)$ & \\
\hline Total & $29(100)$ & $26(100)$ & \\
\hline Menopausal status & & & 0.53 \\
\hline Pre-menopausal & 11 (38) & $12 \quad(46)$ & \\
\hline Post-menopausal & $18 \quad(29)$ & $12 \quad(54)$ & \\
\hline Total & $29(100)$ & $26(100)$ & \\
\hline
\end{tabular}

ER, estrogen receptor; PgR, progesterone receptor.

characteristics between the CT group and the trastuzumabcontaining $(\mathrm{CT}+\mathrm{T})$ group. Clinical complete response (cCR) rates were $6.9 \%$ for NAC without trastuzumab and $46.2 \%$ for NAC with trastuzumab. The pCR rates were $13.8 \%$ for NAC without trastuzumab and $26.9 \%$ for NAC with trastuzumab. Pathological response rates (i.e., quasi-pCR) were $31.0 \%$ for NAC without trastuzumab and $65.4 \%$ for NAC with trastuzumab (Table II).

Changes in the biological markers of HR-/HER2-positive breast cancer. No significant differences with respect to the mean proportion of ER-, PgR-, and Ki-67-positive cells at diagnosis were noted between the $\mathrm{CT}$ and $\mathrm{CT}+\mathrm{T}$ groups. The CT group showed a significantly decreased proportion of ER-positive cells at surgery compared to the proportion prior to NAC (50.9 vs. $73.5 \%, \mathrm{P}=0.02)$ (Table III). On the other hand, no changes were found in the proportion of ER-positive cells at surgery compared to that prior to NAC (80.3 vs. $81.9 \%$, $\mathrm{P}=0.61$ ) for the $\mathrm{CT}+\mathrm{T}$ group (Table III). As noted above, treatment responses were significantly improved, and the tumor reduction ratio was significantly higher in the $\mathrm{CT}+\mathrm{T}$ group than that in the CT group. Consequently, ER-negative cells were more commonly eradicated in the $\mathrm{CT}+\mathrm{T}$ group (Fig. 1).

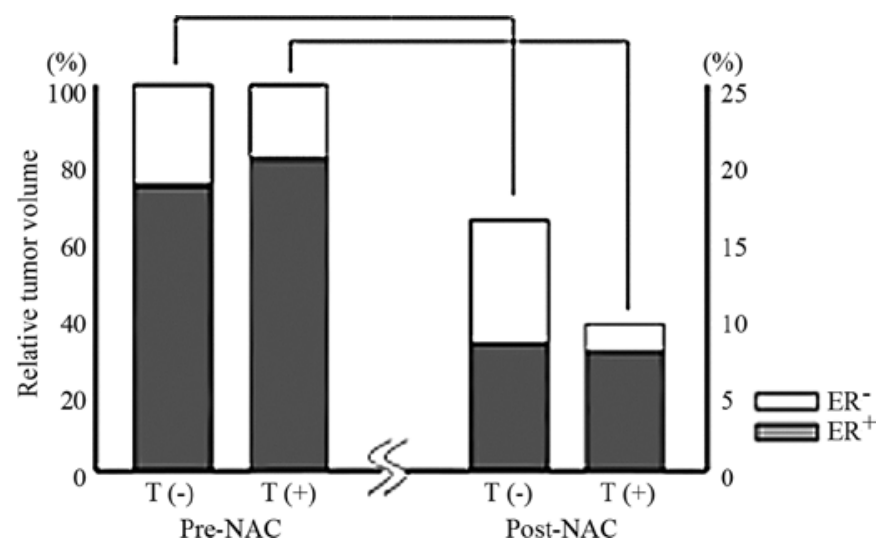

Figure 1. Trastuzumab-containing chemotherapy preferably eradicated ER-negative breast cancer cells compared to chemotherapy alone. The products of the longer and shorter diameters of each tumor are determined as relative tumor volumes, and the means of their products were calculated for each regimen with and without trastuzumab at each time point pre- and post-neoadjuvant chemotherapy (NAC). In each regimen, the tumor volume at pre-NAC was defined as $100 \%$ tumor volume, followed by calculating reduced tumor burdens at post-NAC for each regimen. Proportions of ER-positive cells are shown in the gray area of the columns. Error bars were omitted for improved clarity of the figure. 
Table II. Clinical and pathological responses according to treatment regimens.

\begin{tabular}{|c|c|c|c|}
\hline & $\begin{array}{l}\text { Chemotherapy alone } \\
\qquad(\mathrm{n}=29)\end{array}$ & $\begin{array}{l}\text { Chemotherapy + Trastuzumab } \\
\qquad(\mathrm{n}=26)\end{array}$ & \\
\hline Responses & No. of patients (\%) & No. of patients (\%) & P-value \\
\hline Clinical response & & & 0.002 \\
\hline $\mathrm{cCR}$ & $2 \quad(7)$ & $12 \quad(46)$ & \\
\hline cPR & $24 \quad(83)$ & $11 \quad(42)$ & \\
\hline $\mathrm{cSD}$ & $3 \quad(10)$ & $3 \quad(12)$ & \\
\hline cPD & $0 \quad(0)$ & $0 \quad(0)$ & \\
\hline Total & $29(100)$ & $26(100)$ & \\
\hline Pathological response & & & 0.010 \\
\hline Quasi-pCR & $9 \quad(31)$ & $17 \quad(65)$ & \\
\hline Non-quasi-pCR & $20 \quad(69)$ & $9 \quad(35)$ & \\
\hline Total & $29(100)$ & $26(100)$ & \\
\hline
\end{tabular}

cCR, clinical complete response; cPR, clinical partial response; cSD, clinical stable disease; cPD, clinical progressive disease; quasi-pCR, pCR plus near pCR. pCR, pathological complete response; no residual invasive carcinoma cells in the original tumor and near pCR, marked changes approaching a complete response in only a few remaining invasive carcinoma cells. Two criteria are defined based on the standards of the Japanese Breast Cancer Society (11).

Table III. Changes in biological markers prior to neoadjuvant chemotherapy and at surgery in HER2/HR-positive breast cancer.

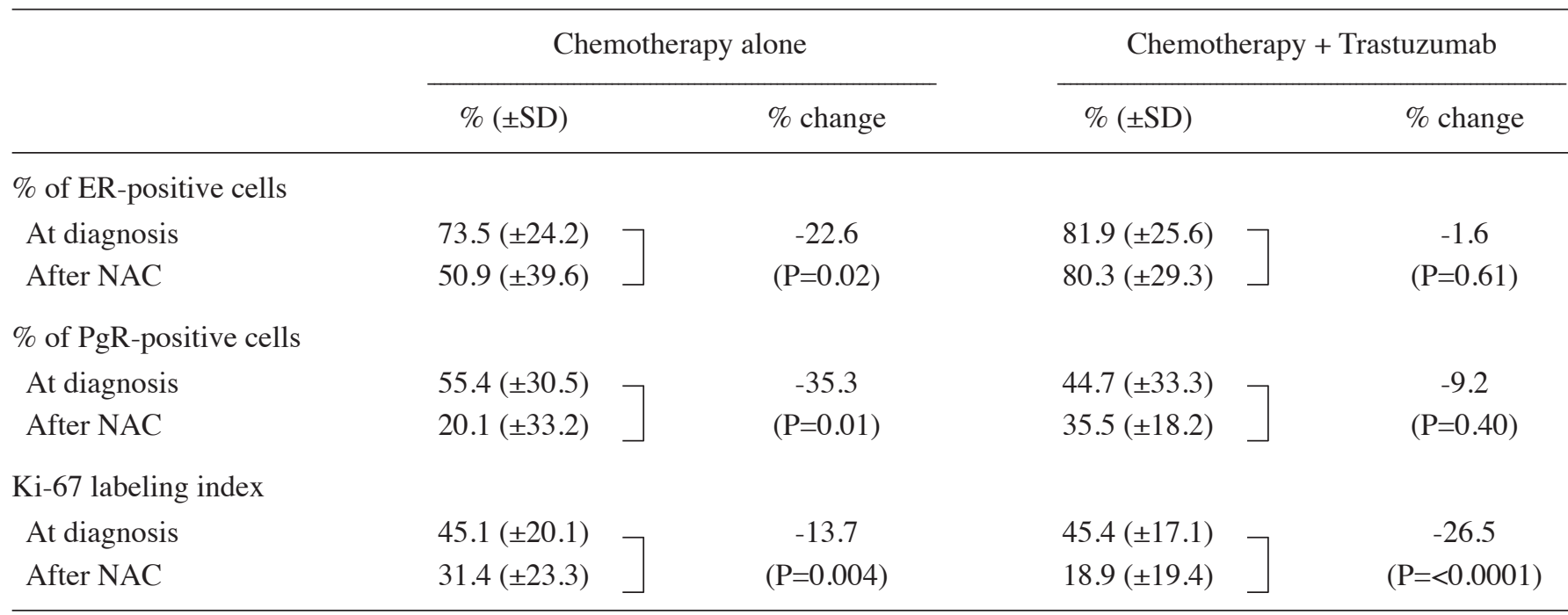

Breast tumors with $10 \%$ or higher proportional positivity for ER, PgR or Ki67 were assessed independently for changes in the proportion from diagnosis to the proportion after NAC. Patients who achieved pCR were excluded from this analysis. Paired Student's t-test was used for statistical analysis. ER, estrogen receptor; PgR, progesterone receptor; SD, standard deviation.

Similar findings were observed for the PgR-positive tumors. In the CT group, the proportion of PgR-positive cells was significantly decreased at surgery compared to the proportion prior to NAC (20.1 vs. $55.4 \%, \mathrm{P}=0.01)$ (Table III). In contrast, in the $\mathrm{CT}+\mathrm{T}$ group, no changes were observed in the proportion of PgR-positive cells in the ER-positive subset at surgery compared to that prior to NAC (35.5 vs. $44.7 \%$, $\mathrm{P}=0.40$ ) (Table III).

Although a significant decrease in Ki-67-positive cells was observed in the two treatment groups, the change in proportion was much greater in the $\mathrm{CT}+\mathrm{T}$ group than the change in proportion in the CT group (-26.5 vs. $-13.7 \%$ ) (Table III). This result suggests that trastuzumab sensitizes proliferative cancer cells to taxane-based cytotoxic chemotherapy.

\section{Discussion}

The present study showed that HER2-positive breast cancers treated with anthracycline-based chemotherapy followed by taxane with trastuzumab showed a higher pathological response rate than those treated with conventional chemotherapy. Moreover, a discrepancy in changes in the proportion 
of ER-, PgR- and Ki-67-positive cells from pre- to post-NAC was noted in the two groups. Through retrospective cellular insight into HER2-positive cancers, we showed direct evidence for a shift in target in eradicated tumor cells from ER-positive to ER-negative by the addition of trastuzumab to taxane-based chemotherapies. This observation was based on the findings of improved objective responses and a significant reduction in total counts of ER-negative HER2-positive cancer cells following treatment with trastuzumab in combination with chemotherapy compared to treatment with chemotherapy alone.

Various molecular and cellular effects of trastuzumab, such as the activation of antibody-dependent cellular cytotoxicity, prevention of shedding of the extracellular domain (ECD) of HER2, inhibition of cell proliferation by preventing HER2-activated intracellular signaling and inhibition of HER2regulated angiogenesis, were previously reported (1,13-15). Trastuzumab administered in combination with conventional types of chemotherapy has been shown to exhibit an additional effect involving improvement of the pCR rate for NACs $(4,5)$. However, eradication of targeted cells by the additional effect of trastuzumab administered in combination with conventional types of chemotherapy has yet to be investigated. In the present study, the additional effect of trastuzumab administered in combination with conventional types of chemotherapy was targeted mainly at ER-negative cells. In addition, PgR-negative and proliferative cells (Ki-67-positive cells) were preferably eradicated with trastuzumab-containing chemotherapies. Trastuzumab is thus considered to sensitize proliferating tumor cells independently of the ER genomic pathway when administered in combination with taxane-based chemotherapies.

ER genomic pathway-independent proliferating tumor cells are considered to be responsible for disease recurrence and resistance to various types of chemotherapy. The CSC hypothesis similarly asserts that disease recurrence and treatment resistance to various types of chemotherapy and irradiation can be attributed to this type of tumor cell. Thus, ER pathway-independent proliferating cells may merge with treatment-resistant tumor cells, such as CSCs. Concerning the association between CSCs and the clinicopathological characteristics of breast cancer, ALDH-1-positive breast cancer cells, which are putative breast CSCs, are more likely to be ER-negative, Ki-67-positive and HER2-positive $(16,17)$. Moreover, the proportion of cells with these markers is closely related with poor prognosis in breast cancer. $\mathrm{Li}$ et al showed that tumorigenic cancer cells, which are phenotypically determined as CD44-positive/CD24-negative, are intrinsically resistant to conventional chemotherapy. These authors also showed that a dual inhibitor of HER1/HER2, lapatinib, regulates the proportion of tumorigenic cells in a tumor (18). Thus, the CSC hypothesis has been accepted as clinically relevant and is applied to the treatment of breast cancer. Previous findings and the results of the present study indicate that further investigation of the molecular rationale of molecular-targeting therapies used to overcome chemotherapy resistance in breast cancer is required.

The decision to define quasi-pCR by grouping bona fide pCR and near-pCR in this study was justified by the evidence that following pre-operative chemotherapy quasi-pCR predicts favorable disease-free survival (12). This criterion was applied to determine the type of tumor cell that was likely to be eradicated by the addition of trastuzumab to taxane-based chemotherapies. Findings showed the pathological response rate or quasi-pCR to be $65.4 \%$ in the $\mathrm{CT}+\mathrm{T}$ group. These data are comparable with those of previous reports involving NAC with or without trastuzumab (4,19-21), confirming our analysis.

In conclusion, the insight gained from the present study may provide a biological rationale of the additional clinical efficacy of trastuzumab administered in combination with conventional types of chemotherapy. However, the molecular mechanism for this shift in target from ER-positive to ER-negative breast cancer cells in the treatment of trastuzumab-containing chemotherapies should be investigated. Furthermore, prospective studies testing the hypothesis that trastuzumab sensitizes treatment-resistant breast cancer cells or those cells responsible for recurrence to conventional chemotherapies are warranted.

\section{Acknowledgements}

We thank the staff at the Department of Clinical Pathology of Kumamoto City Hospital for their technical assistance.

\section{References}

1. Hudis CA: Trastuzumab - mechanism of action and use in clinical practice. N Engl J Med 357: 39-51, 2007.

2. Slamon DJ, Leyland-Jones B, Shak S, et al: Use of chemotherapy plus a monoclonal antibody against HER2 for metastatic breast cancer that overexpresses HER2. N Engl J Med 344: 783-792, 2001.

3. Marty M, Cognetti F, Maraninchi D, et al: Randomized phase II trial of the efficacy and safety of trastuzumab combined with docetaxel in patients with human epidermal growth factor receptor 2-positive metastatic breast cancer administered as first-line treatment: the M77001 study group. J Clin Oncol 23: 4265-4274, 2005.

4. Buzdar AU, Ibrahim NK, Francis D, et al: Significantly higher pathologic complete remission rate after neoadjuvant therapy with trastuzumab, paclitaxel, and epirubicin chemotherapy: results of a randomized trial in human epidermal growth factor receptor 2-positive operable breast cancer. J Clin Oncol 23: 3676-3685, 2005

5. Gianni L, Eiermann W, Semiglazov V, et al: Neoadjuvant chemotherapy with trastuzumab followed by adjuvant trastuzumab versus neoadjuvant chemotherapy alone, in patients with HER2-positive locally advanced breast cancer (the NOAH trial): a randomised controlled superiority trial with a parallel HER2-negative cohort. Lancet 375: 377-384, 2010.

6. Al-Hajj M, Wicha MS, Benito-Hernandez A, Morrison SJ and Clarke MF: Prospective identification of tumorigenic breast cancer cells. Proc Natl Acad Sci USA 100: 3983-3988, 2003.

7. Liu S, Dontu G and Wicha MS: Mammary stem cells, selfrenewal pathways, and carcinogenesis. Breast Cancer Res 7: 86-95, 2005.

8. Kai K, Nishimura R, Arima N, Miyayama H and Iwase H: p53 expression status is a significant molecular marker in predicting the time to endocrine therapy failure in recurrent breast cancer: a cohort study. Int J Clin Oncol 11: 426-433, 2006.

9. Therasse P, Arbuck SG, Eisenhauer EA, et al: New guidelines to evaluate the response to treatment in solid tumors. European Organization for Research and Treatment of Cancer, National Cancer Institute of the United States, National Cancer Institute of Canada. J Natl Cancer Inst 92: 205-216, 2000.

10. Kurosumi M, Akashi-Tanaka S, Akiyama F, et al: Histopathological criteria for assessment of therapeutic response in breast cancer (2007 version). Breast Cancer 15: 5-7, 2008.

11. Kuroi K, Toi M, Tsuda H, Kurosumi M and Akiyama F: Issues in the assessment of the pathologic effect of primary systemic therapy for breast cancer. Breast Cancer 13: 38-48, 2006.

12. Toi M, Nakamura S, Kuroi K, et al: Phase II study of preoperative sequential FEC and docetaxel predicts of pathological response and disease-free survival. Breast Cancer Res Treat 110: 531-539, 2008. 
13. Molina MA, Codony-Servat J, Albanell J, Rojo F, Arribas J and Baselga J: Trastuzumab (Herceptin), a humanized anti-Her2 receptor monoclonal antibody, inhibits basal and activated Her2 ectodomain cleavage in breast cancer cells. Cancer Res 61: 4744-4749, 2001

14. Gennari R, Menard S, Fagnoni F, et al: Pilot study of the mechanism of action of preoperative trastuzumab in patients with primary operable breast tumors overexpressing HER2. Clin Cancer Res 10: 5650-5655, 2004.

15. Nahta R and Esteva FJ: HER2 therapy: molecular mechanisms of trastuzumab resistance. Breast Cancer Res 8: 215, 2006.

16. Ginestier C, Hur MH, Charafe-Jauffret E, et al: ALDH1 is a marker of normal and malignant human mammary stem cells and a predictor of poor clinical outcome. Cell Stem Cell 1: 555-567, 2007.

17. Morimoto K, Kim SJ, Tanei T, et al: Stem cell marker aldehyde dehydrogenase 1-positive breast cancers are characterized by negative estrogen receptor, positive human epidermal growth factor receptor type 2, and high Ki67 expression. Cancer Sci 100: 1062-1068, 2009
18. Li X, Lewis MT, Huang $\mathrm{J}$, et al: Intrinsic resistance of tumorigenic breast cancer cells to chemotherapy. J Natl Cancer Inst 100: 672-679, 2008

19. Paluch-Shimon S, Wolf I, Goldberg H, et al: High efficacy of pre-operative trastuzumab combined with paclitaxel following doxorubicin \& cyclophosphamide in operable breast cancer. Acta Oncol 47: 1564-1569, 2008

20. Chumsri S, Jeter S, Jacobs LK, et al: Pathologic complete response to preoperative sequential doxorubicin/cyclophosphamide and single-agent taxane with or without trastuzumab in stage II/III HER2-positive breast cancer. Clin Breast Cancer 10: 40-45, 2010.

21. Chen XS, Wu JY, Huang O, et al: Molecular subtype can predict the response and outcome of Chinese locally advanced breast cancer patients treated with preoperative therapy. Oncol Rep 23: $1213-1220,2010$ 